\title{
Strong Adsorption of Phosphorus by Zn-Al-LDO-Activated Banana Biochar: An Analysis of Adsorption Efficiency, Thermodynamics and Internal Mechanisms
}

\author{
Anyu Li ${ }^{\mathrm{a}, \mathrm{b}}$, Hua Denga ${ }^{\mathrm{a}, \mathrm{b}^{* *}}$, Yuqing Wu ${ }^{\mathrm{a}, \mathrm{b}}$, Chenghui Ye ${ }^{\mathrm{a}, \mathrm{b}}$, Yanhong Jiang ${ }^{\mathrm{a}, \mathrm{b}^{* *}}$ \\ Anyu Li: College of Environment and Resources, Guangxi Normal University, Guilin 541006, China. \\ Hua Deng: College of Environment and Resources, Guangxi Normal University, Guilin 541006, China. \\ Yuqing Wu: College of Environment and Resources, Guangxi Normal University, Guilin 541006, \\ China. \\ Chenghui Ye: College of Environment and Resources, Guangxi Normal University, Guilin 541006, \\ China. \\ Yanhong Jiang: College of Environment and Resources, Guangxi Normal University, Guilin 541006, \\ China.
}

\footnotetext{
*Corresponding author: The University Key Laboratory of Karst Ecology and Environmental Change of Guangxi Province,

College of Environment and Resources, Guangxi Normal University, Guilin 541006, China.

E-mail address: denghua@mailbox.gxnu.edu.cn (H. Deng).
} 


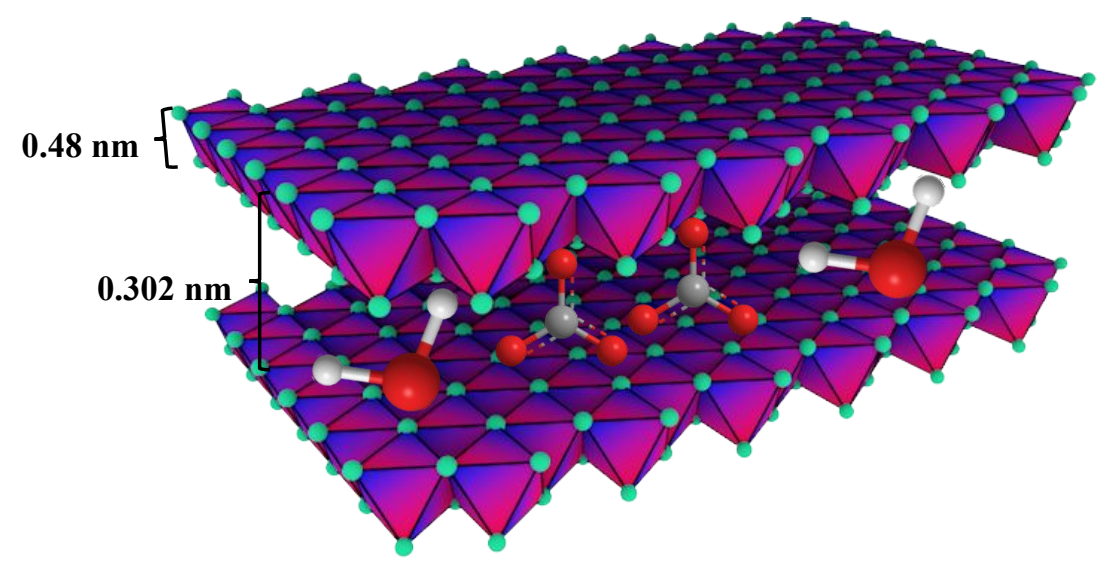

Figure S1 The apparent structure of Zn-Al-LDO. 


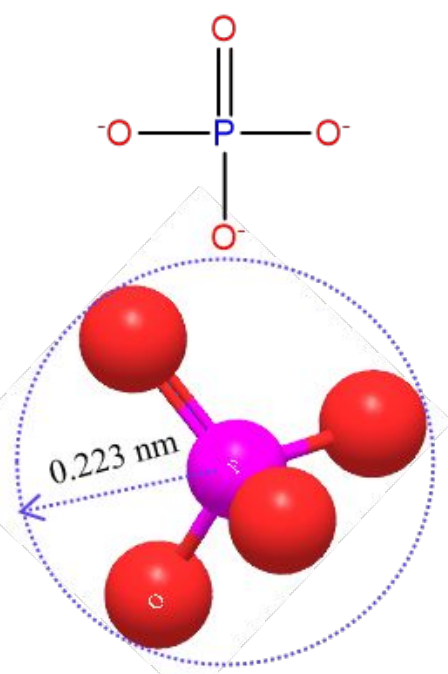

Figure S2 The crystal radius of phosphate. 


\section{Information of the drugs}

Potassium dihydrogen phosphate $\left(\mathrm{KH}_{2} \mathrm{PO}_{4}, \mathrm{AR}\right)$, sulfuric acid $\left(\mathrm{H}_{2} \mathrm{SO}_{4}, \mathrm{AR}\right)$, ascorbic acid $\left(\mathrm{C}_{6} \mathrm{H}_{8} \mathrm{O}_{6}\right.$, AR), ammonium molybdate $\left(\mathrm{H}_{8} \mathrm{MoN}_{2} \mathrm{O}_{4}, \mathrm{AR}\right)$ and potassium persulfate $\left(\mathrm{K}_{2} \mathrm{~S}_{2} \mathrm{O}_{8}, A R\right)$ were obtained from Sinopharm Chemical Reagent Co., Ltd., and did not undergo further purification 